\title{
Comparison of Two Different Calculation Methods of Fractional Vegetation Cover
}

\author{
Liao kaitao $^{1}$ \\ ${ }^{1}$ Jiangxi Academy of Water Science and Engineering, 330029 Nanchang Jiangxi, China \\ Jiangxi Normal University, College of Geography and Environment, 330022 Nanchang Jiangxi, China
}

\begin{abstract}
Fractional Vegetation Cover (FVC) is an important parameter for soil erosion equation. The Fractional Vegetation Cover of typical soil erosion county in red soil region of South China was calculated by parameter revision method (PRM) and fusion method (FM).The results of the two methods were compared from two parts of FVC level and 24 half-months FVC. The results show that the FVC level calculated by PRM was concentrated in high level, and that calculated by FM is mainly in medium-high level and high level. The time variation of FVC in 24 half-months calculated by the two methods was the same, but the majority of FVC value calculated by PRM was higher than that calculated by FM. It is better to choose FM method to calculate FVC in estimating soil erosion.
\end{abstract}

\section{Introduction}

Fractional Vegetation Cover (FVC) is not only an indicator of surface vegetation status, but also an important index to evaluate regional hydrological, ecological and environmental changes [1-3]. FVC is one of the important factors affecting soil erosion, which can directly reflect the change of vegetation coverage or the ability of vegetation measures to control soil erosion [4], and it is an important parameter of Chinese Soil Loss Equation (CSLE) [5]. With the rapid development of remote sensing technology, using satellite image data to estimate regional FVC has become the main method [68]. The Technical Regulations on Dynamic Monitoring of Regional Soil Erosion (Trial), which promulgated by Monitoring Centre of Soil and Water Conservation, Ministry of Water Resources of the People's Republic of China in 2020, required local government to use Landsat satellite data and MODIS-NDVI data to calculate regional FVC, and provided two calculation methods, one is called fusion method (FM), the other is called parameter revision method (PRM).

At present, most regions of China used the parameter revision method to estimate FVC. However, the data parameter revision method used was from 2011, cannot accurately express the current situation, because the land cover had changed a lot. In order to compare the difference between the fusion method and the parameter revision method, Wuning County of Jiangxi Province was taken as the study area, two different methods were used to estimate $\mathrm{FVC}$, and the results were compared and analyzed. This hopes to provide reliable basic parameters for improving the calculation accuracy of annual soil erosion dynamic monitoring in China and provide beneficial enlightenment for soil erosion research.

\section{Materials and Methods}

\subsection{Study area}

Wuning County is located in the northwest of Jiangxi Province, and in the middle and lower reaches of the Yangtze River, within a longitude of $114^{\circ} 28^{\prime} \mathrm{E}$ to $115^{\circ} 27^{\prime} \mathrm{E}$ and a latitude of $28^{\circ} 52^{\prime} \mathrm{N}$ to $29^{\circ} 35^{\prime} \mathrm{N}$, occupying 3507 square kilometres. The study area is in the southern edge of the middle subtropical zone, belongs to the typical subtropical humid monsoon climate zone with an annual average of $1488 \mathrm{~mm}$, and the annual average temperature is about $16.6^{\circ} \mathrm{C}$.

The main land use type of study area are forest land, agricultural land, water. The forest land area is 2687.14 $\mathrm{km}^{2}, 76.64 \%$ of the total land use area, the agricultural land area is $346.16 \mathrm{~km}^{2}, 97 \%$ of the total land use area, and the water area is $279.67 \mathrm{~km} \mathrm{~km}^{2}, 9.86 \%$ of the total land use area. The area of soil erosion is $222.04 \mathrm{~km}^{2}$, accounts for $6.33 \%$ of the total land use area, all of which are water erosion. Among them, the light erosion area is $198.05 \mathrm{~km}^{2}$, $89.20 \%$ of the total area of soil erosion. The moderate erosion area is $16.08 \mathrm{~km}^{2}, 7.24 \%$ of the total area of soil erosion.

\subsection{Remote image}

The data used in the study include Landsat OLI (20172019), MODIS-NDVI (2017-2019), land use (2019) and correction coefficient image (2011).

The resolution of Landsat OLI image is $30 \mathrm{~m}$, and there are 9 bands in total, of which 4 and 5 bands are Red band and NIR band respectively for NDVI calculation, and 9 band is cirrus band for cloud detection. The resolution of

\footnotetext{
* Corresponding author: Liaokaitao@jxnu.edu.cn
} 
MODIS-NDVI image is 250m, revisit period is 16 day, 23 images per year. The radiometric calibration, geometric correction, cloud / snow / shadow mask, mosaic and NDVI calculation of Landsat OLI images are all completed on the Google Earth Engineer (GEE) cloud platform [9].

The land use data was based on visual interpretation of GF-2 image, which resolution is about $2 \mathrm{~m}$. Correction coefficient image was supplied by Ministry of Water Resources of the People's Republic of China.

\subsection{Image fusion method (FM)}

Based on land use data, the half-month frequency vegetation index series of all kinds of pure pixels were determined:

$$
V_{M}(t)=\frac{1}{3 \times N} \sum_{y=k}^{k+2} \sum_{n=1}^{N} N D V I(t, y, n)
$$

Where $V_{M}(t)$ is a certain category of Multi-year NDVI mean value, $N$ is the number of pure pixels, $y$ is the year, $k$ is the starting year of the first three years of the monitoring year.

Then based on function 2, process Landsat OLI NDVI data with MODIS-NDVI data, obtained 23 NDVI images of $30 \mathrm{~m}$.

$$
V_{H}\left(t_{i}\right)=V_{M}\left(t_{i}\right)+\frac{\sum_{j=1}^{n}\left[\omega\left(t_{i}, t_{j}\right)\left(V_{T}\left(t_{j}\right)-V_{M}\left(t_{j}\right)\right)\right]}{\sum_{j=1}^{n} \omega\left(t_{i}, t_{j}\right)}
$$

Where $V_{H}\left(t_{i}\right)$ is the result of image fusion, $V_{M}\left(t_{i}\right)$ is the MODIS-NDVI multi-year mean value sequence corresponding to each pixel, $V_{T}\left(t_{j}\right)$ is the Landsat NDVI value of each pixel at the certain time, $\mathrm{n}$ is the number of Landsat OLI images, $t_{i}$ is the Julian day of MODIS-NDVI image, $t_{j}$ is the Julian day of Landsat OLI image, $\omega\left(t_{i}, t_{j}\right)$ is the weight of Landsat OLI image.

Then based on function 3 , converting NDVI data to FVC :

$$
F V C=\frac{N D V I-N D V I_{\min }}{N D V I_{\max }-N D V I_{\min }}
$$

Where $N D V I_{\max }$ is the NDVI value of pure vegetation coverage, and $N D V I_{\min }$ is the NDVI value of pure bare soil. In this paper, according to the cumulative frequency table of NDVI gray distribution in the whole image, the upper and lower cumulative frequencies in the confidence interval of $5 \%$ and $95 \%$ were selected as $N D V I_{\min }$ and $N D V I_{\max }$, respectively.

\subsection{Parameter revision method (PRM)}

Firstly, used MODIS-NDVI data obtain $23 F V C_{L}$ images by function 3 . Then based on function 4 , obtain $23 F V C_{H}$ images of $30 \mathrm{~m}$.

$$
F V C_{H}=F V C_{L} \times a
$$

Where $a$ is the correction coefficient image supplied by Ministry of Water Resources of the People's Republic of China.

In CSLE model, it need $24 \mathrm{FVC}$ images to calculate soil erosion, but MODIS-NDVI only have 23 images a year, so take the average value of the 8th and 9th FVC image as the new 9th FVC image.

Use the same method, the FVC values of the 24 in 2017, 2018 and 2019 were calculated, then average the data of three years as the finally FVC results.
The FVC is divided into five levels: $0-30 \%$ for low level, $30-45 \%$ for medium-low level, $45-60 \%$ for medium level, $60-75 \%$ for medium-high level and $75-100 \%$ for high level.

\section{Results and Discussion}

FVC is related to vegetation, so we compare the FVC of forest land, grassland and orchard calculated by the two different methods. The vegetation in the study area grows best in September, so select the 17th period FVC for statistical analysis.

\subsection{FVC Level analysis}

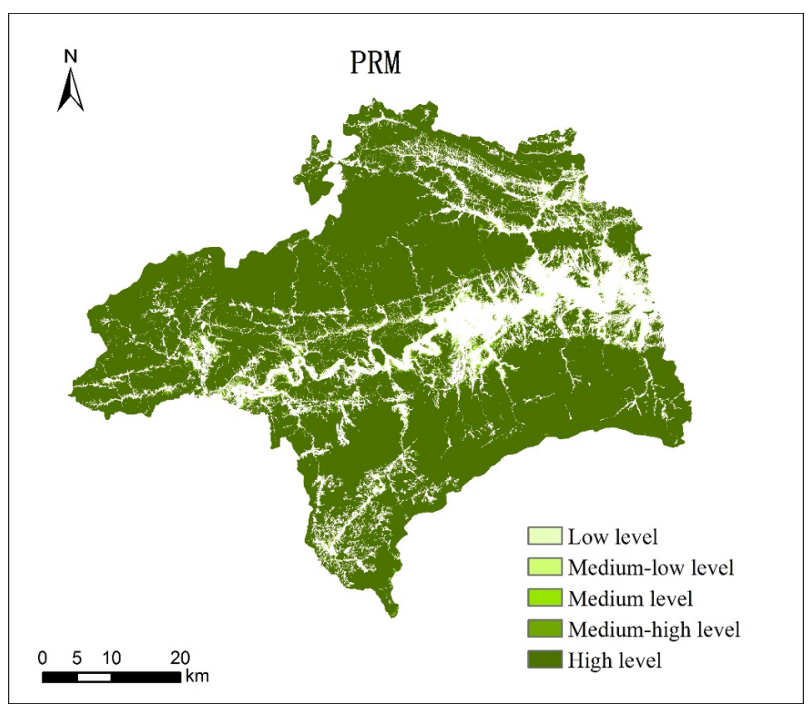

Fig. 1. The FVC level calculated by PRM

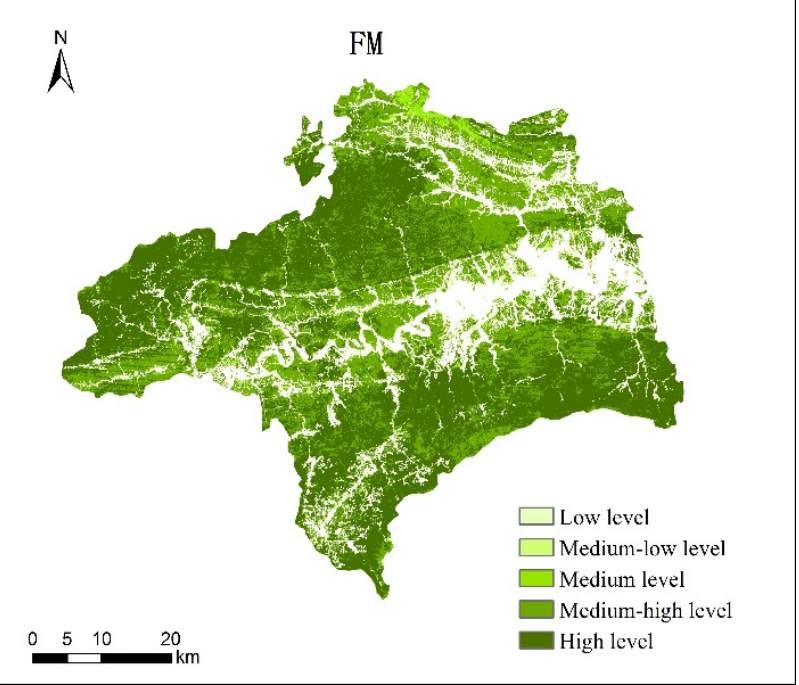

Fig. 2. The FVC level calculated by FM Compared with figure 1 and Figure 2, it can be seen that the FVC level calculated by PRM was mainly concentrated in high level, and the other several levels of FVC were basically invisible. But the FVC level calculated by FM was mianly distributed alternately in high level and medium-high level.

Table 1 shows that the FVC of Wuning County calculated by the two different methods were all mainly 
in high level. In the low level, the area calculated by PRM is $39.96 \mathrm{~km}^{2}$, and the area calculated by FM is $21.37 \mathrm{~km}^{2}$, which is $18.59 \mathrm{~km}^{2}$ smaller than that PRM. In the mediumlow level, the area calculated by the PRM is $9.93 \mathrm{~km}^{2}$, the area calculated by FM is $29.73 \mathrm{~km}^{2}$, which is $19.80 \mathrm{~km}^{2}$ smaller than that PRM. In the medium level, the area calculated by PRM is $24.04 \mathrm{~km}^{2}$, the area calculated by the FM is $106.34 \mathrm{~km}^{2}$, which is $82.30 \mathrm{~km}^{2}$ larger than that PRM. In the medium-high level, the area calculated by PRM is $69.24 \mathrm{~km}^{2}$, the area calculated by the FM is $1099.78 \mathrm{~km}^{2}$, which is $1030.54 \mathrm{~km}^{2}$ larger than that PRM. In the high level, the area calculated by PRM is $2626.66 \mathrm{~km}^{2}$, and the area calculated by the FM is $1512.63 \mathrm{~km}^{2}$, which is $1114.03 \mathrm{~km}^{2}$ smaller than that PRM. Compared the results of FM and PRM, the FVC calculated by PRM is higher and concentrated in the high value area.

Table 1. The area of different FVC level

\begin{tabular}{|c|c|c|c|}
\hline \multicolumn{2}{|c|}{ FVC Level } & \multirow{2}{*}{$\frac{\text { FM }}{21.37}$} & \multirow{2}{*}{$\begin{array}{c}\text { PRM } \\
39.96\end{array}$} \\
\hline Iow & Area $\left(\mathrm{km}^{2}\right)$ & & \\
\hline LOW & Percentage（\%) & 0.77 & 1.44 \\
\hline \multirow{2}{*}{ Medium-low } & Area $\left(\mathrm{km}^{2}\right)$ & 29.73 & 9.93 \\
\hline & Percentage (\%) & 1.07 & 0.36 \\
\hline \multirow{2}{*}{ Medium } & Area $\left(\mathrm{km}^{2}\right)$ & 106.34 & 24.04 \\
\hline & Percentage (\%) & 3.84 & 0.87 \\
\hline \multirow{2}{*}{ Medium-high } & Area $\left(\mathrm{km}^{2}\right)$ & 1099.78 & 69.24 \\
\hline & Percentage (\%) & 39.71 & 2.50 \\
\hline \multirow{2}{*}{ High } & Area $\left(\mathrm{km}^{2}\right)$ & 1512.63 & 2626.66 \\
\hline & Percentage (\%) & 54.61 & 94.83 \\
\hline \multicolumn{2}{|c|}{ Total $\left(\mathrm{km}^{2}\right)$} & 2769.85 & 2769.85 \\
\hline
\end{tabular}

\subsection{Analysis of FVC in $\mathbf{2 4}$ half months}

Figure 3 shows that FVC of orchard in 24 half-months calculated by PRM is 0.33 to 1 , the lowest is 0.33 in the 4th half-month, and the FVC reach the highest 1 from 10th to 20th half month. The FVC of orchard in 24 half-months calculated by FM ranged from 0.42 (the 5 th half-month) to 0.93 (the 13th half-months). From the 6th half-month to the 23rd half-month, the vegetation coverage calculated by PRM is higher than that calculated by FM.

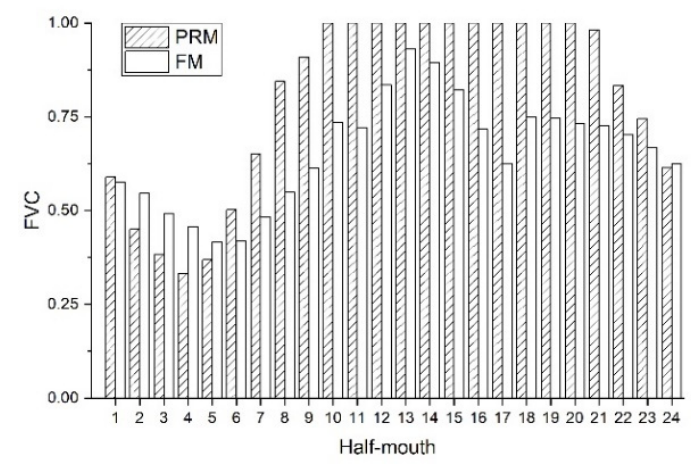

Fig. 3. FVC of orchard in 24 half-months
Figure 4 shows that FVC of forest land in 24 halfmonth calculated by PRM is 0.83 to 1 , the lowest is in the second half-month, and the FVC from the 8th half-month to the 24th half-month is 1 . The FVC calculated by FM ranged from 0.76 (the 9th half-month) to 0.98 (the 5 th half-month). From the 8th half-month to the 24th halfmonth, the FVC calculated by PRM is higher than that calculated by FM.

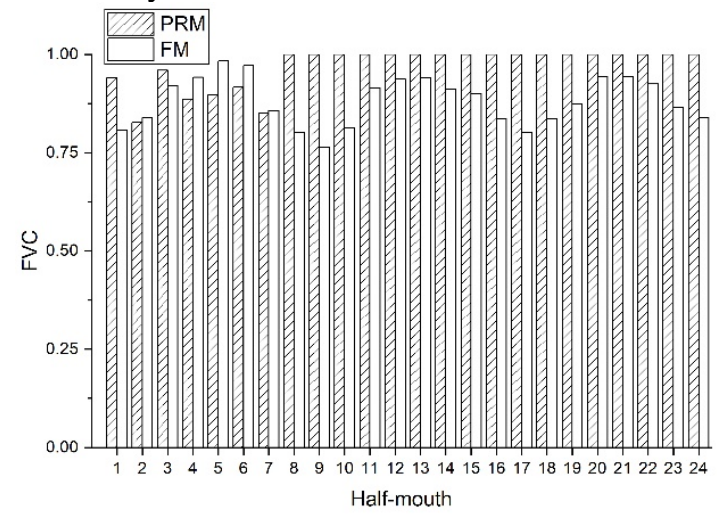

Fig. 4. FVC of forest land in 24 half-months

Figure 5 shows that the FVC of grassland calculated by PRM in 24 half-month is 0.34 to 1 , the lowest in the 5th half-month, and the FVC from the 10th to the 19th half-month is 1 . The FVC of grassland calculated by FM in 24 half-month is 0.36 (the 4th half-month) and 1 (12th half-month). From the 6th half-month to the 24th halfmonth, the FVC calculated by PRM is higher than that calculated by FM.

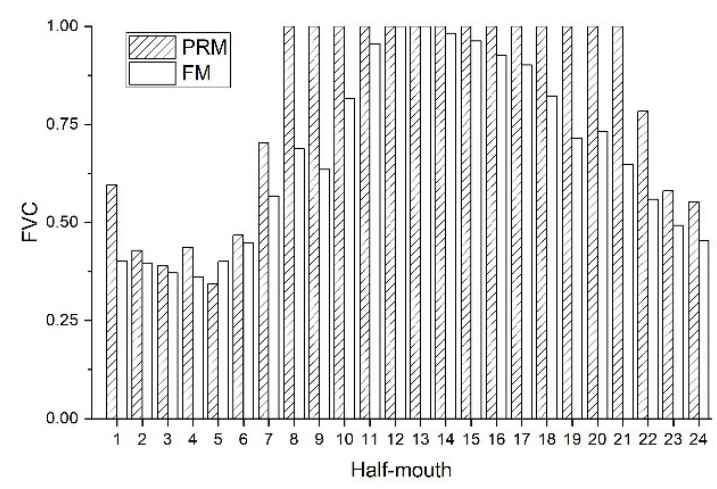

Fig. 5. FVC of grassland in 24 half-months

Generally speaking, the minimum value of the FVC calculated by PRM generally appears in the 4th or 5th half-month. The maximum value is 1 , and it always begins to appear in the 8th or 10th half-month, and often lasts to about the 20th half-month. The maximum value of FVC calculated by FM is also 1, but only appear in few half-mouth. The maximum value usually occurs in summer or autumn, and the overall value is lower than that calculated by PRM. The overall trend of the two methods is consistent.

\section{Conclusions}

In this paper, a typical red soil erosion county in Jiangxi Province, South China was taken as the study area. The PRM method and FM method were used to calculate FVC 
of Wuning County. Compared the difference between the two methods in FVC classification level and 24 halfmouths. The FVC level calculated by PRM was mainly in high level, while FVC level calculated by FM was mainly distrubuted in medium-high level and high level. The time variation of FVC in 24 half-months calculated by the two methods was the same. The minimum value of the FVC often appeared in 4th or 5th half-mouth, and the maximum value always began to appear in the 8th or 10th half-month. The FVC in summer was the highest during the year. No matter the Vegetation types were, the FVC calculated by PRM method was higher than that calculated by ME method. It is better to choose FM method to calculate FVC in estimating soil erosion.

\section{Acknowledgments}

The study was supported by the Water Conservancy Science and Technology Project of Jiangxi Province (NO. 202124ZDKT25，202022YBKT20， 201922ZDKT08, 202123YBKT16, 201821ZDKT18) and the National Natural Science Foundation of China(NO.41967011) .

\section{References}

1. Zhang J , Zhang Z, Chen J, et al. Estimating soil salinity with different fractional vegetation cover using remote sensing $[\mathrm{J}]$. Land Degradation and Development, 2020.

2. Wu D, Hao W, Zhao X, et al. Evaluation of Spatiotemporal Variations of Global Fractional Vegetation Cover Based on GIMMS NDVI Data from 1982 to 2011[J]. Remote Sensing, 2014, 6(5):4217-4239.

3. Wang $\mathrm{X}$, Jia $\mathrm{K}$, Liang $\mathrm{S}$, et al. Fractional Vegetation Cover Estimation Method Through Dynamic Bayesian Network Combining Radiative Transfer Model and Crop Growth Model[J]. IEEE Transactions on Geoscience \& Remote Sensing, 2016, 54(12):7442-7450.

4. Yang, Xihua. Deriving RUSLE cover factor from time-series fractional vegetation cover for hillslope erosion modelling in New South Wales[J]. Soil Research, 2014, 52(3):253-261.

5. Meusburger K, Konz N, Schaub M, et al. Soil erosion modelled with USLE and PESERA using QuickBird derived vegetation parameters in an alpine catchment $[\mathrm{J}]$. International Journal of Applied Earth Observation and Geoinformation, 2010, 12(3):208215.

6. Zhan Y, Meng Q, Wang C, et al. Fractional vegetation cover estimation over large regions using GF-1 satellite data[J]. Proceedings of SPIE - The International Society for Optical Engineering, 2014, 9260.

7. Zheng Z, Yuan Z, Zhao Y, et al. Monitoring and dynamic analysis of fractional vegetation cover in southwestern China over the past 15 years based on
MODIS data[J]. Remote Sensing for Land \& Resources, 2017.

8. Zhou Z, Yang Y, Chen B . Estimating Spartina alterniflora fractional vegetation cover and aboveground biomass in a coastal wetland using SPOT6 satellite and UAV data[J]. Aquatic Botany, 2017, 144:38-45.

9. Vermeulen L M, Munch Z, Palmer A . Fractional vegetation cover estimation in southern African rangelands using spectral mixture analysis and Google Earth Engine[J]. Computers and Electronics in Agriculture, 2021, 182(12):105980. 